\title{
Case Report: Thoracic Spinal Schwannoma Simulating Lumbar Sciatica-like Pain: A Case Report and Review of Literature
}

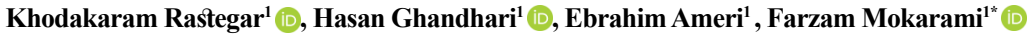 \\ 1. Bone and Joint Reconstruction Research Center, Shafa Orthopedic Hospital, Iran University of Medical Sciences, Tehran, Iran
}

\begin{tabular}{|c|c|}
\hline \multirow{3}{*}{ 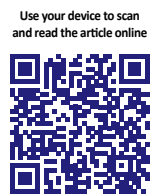 } & Coftat - Rastegar Kh Ghandhari H Ameri E Mokarami E Thoracic Spinal Schwannoma Simulatino Lumbar Sciatica-like \\
\hline & $\begin{array}{l}\text { pain: A Case Report and Review of Literature. Journal of Research in Orthopedic Science. 2021; 8(1):43-50. http://dx.doi. } \\
\text { org/10.32598/JROSJ.8.1.167.1 }\end{array}$ \\
\hline & doif: http://dx.doi.org/10.32598/JROSJ.8.1.167.1 \\
\hline
\end{tabular}

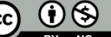

Article info:

Received: 10 Dec 2020

Revised: 15 Dec 2020

Accepted: 05 Jan 2021

Available Online: 01 February 2021

Keywords:

Cord compression, Schwannoma, Tract pain, Sciatica

\begin{abstract}
A B S T RA C T
A 45-year-old man presented to our facility with predominantly Sciatica-like leg pain and lower extremity motor weakness, who did not get relief despite undergoing two consecutive lumbar surgeries for suspected lumbar disc herniation. Medical history, physical findings, and a magnetic resonance imaging scan revealed thoracic cord tumor as the underlying disease. Our patient had complete resolution of his back and leg pain following surgical resection of the thoracic Schwannoma. Thoracic cord compression often results in diffuse pain and myelopathic symptoms caused by the irritation of ascending spinothalamic tract, which causes a vague and burning pain that should be differentiated from nerve root lesions and can be the first presentation of a thoracic cord lesion.
\end{abstract}

\section{Introduction}

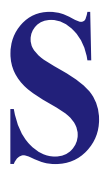

ymptomatic patients with thoracic spinal cord compression frequently present with midline back pain and myelopathy; therefore, sciatica-like symptoms and radiculopathy of lower extremities are not the main or first complaints [1, 2]. However, Lhermitte's sign, neurogenic claudication with positionally dependent weakness, flaccid paraplegia, and chronic abdominal pain can be the unusual manifestations of this pathology $[1,3]$.
Although rare, sciatica has been reported due to compressive lesions of the spinal cord at the cervical or thoracic level [4-7].

Burning pain from cord compression usually originates from the irritation of the ascending spinothalamic tracts below the level of the lesion and presents as a vague and diffuse pain with no dermatomal distribution; hence, it is called sciatica-like pain $[4,8,9]$.

Therefore, attributing a sciatica pain just to a lumbar insult would be an oversimplification and can lead to misdiagnosis and neglecting more important differential

\footnotetext{
* Corresponding Author:

Farzam Mokarami, MD.

Address: Bone and Joint Reconstruction Research Center, Shafa Orthopedic Hospital, Iran University of Medical Sciences, Tehran, Iran

Phone: +98 (21) 33542001

E-mail:farzam_m2001@yahoo.com
} 
diagnoses, namely spinal and extraspinal lesion, cervical myelopathy, multiple sclerosis, and other spinal cord or even intracranial pathologies $[1,5,10,11]$. We report a case of sciatica-like pain caused by a thoracic spinal cord tumor, which complicated the diagnosis and treatment.

\section{Case Presentation}

A 45-year-old man was admitted to our clinic, who had undergone two surgical procedures for lumbar disc herniation and stenosis in the last 20 days. The patient reported back pain and right side dominant sciatica-like pain in both legs and neurogenic claudication for the past 6 months, and also expressed slight bilateral numbness in both legs and feet, despite adequate conservative treatments, including physiotherapy and oral medication, with no notable improvement.

Neurological examination before the index surgery (as documented in his medical records) showed bilateral motor weakness in L3 to L5 spinal segments (grade 4); there was no hyperreflexia or any abnormal gait. The straight leg raising test was negative and he denied loss of bladder or bowel control.

The Magnetic Resonance Imaging (MRI) examination of the lumbar spine showed that there was a loss of disc height and protrusion at L3-L4 level and right-sided disc herniation with mild lateral recess stenosis at L4-L5 level.

Due to the failure of non-operative treatments, the surgeon decided to proceed with surgical decompression of L4-L5 intervertebral level based on MRI findings by performing L4 laminotomy and L4-L5 discectomy under the diagnosis of L4-L5 stenosis and disc protrusion. After the operation, no major differences in the patient's symptoms were noticed, and in the coming days (gradually after 2 weeks), muscle forces of the lower extremi- ties started to decline to grade 1, and bowel and bladder control were lost. The second MRI added no more information, and then surgical exploration to detect pathology (probable hematoma or incomplete release) was undertaken, and more extensive decompression and L3 to L5 fusion and instrumentation were done and the patient was sent to our center for more evaluations.

We conducted a thorough examination, which revealed motor weakness grade 1 (muscle contraction only) in lower extremities, hyperreflexia and upward Babinski reflexes, and clonus in both ankles. Bladder control was impaired and an indwelling catheter was used and cervical and thoracic evaluation was performed by MRI because upper motor neuron disorder was not explained by the lumbar MRI findings. The thoracic MRI demonstrated an intradural tumor at the L5 level, iso-signal intensity on T1-weighted, and hyper signal intensity on T2-Weighted Images (WI) that pushed away from the thoracic cord to right on axial MRI view.

Considering progressive neurologic deficit, urgent tumor resection was done through a posterior T5 to T7 laminectomy and durotomy, and an Intradural Extramedullary (IE) solid mass $(17 \times 7 \mathrm{~mm})$ was excised and removed; thus, the spinal cord returned to the central position. The pathology report proved that the specimen was a Schwanomma. The patient's sciatica-like and back pain immediately disappeared following the operation (Figures 1 and 2).

One month postoperatively, the patient still depended on bowel and bladder aid devices but spasms and hyperreflexia decreased to an extent that muscle contractions can be done more voluntarily and muscle strength grade 3 appeared in lower extremities. The patient gave consent for his information to be presented in this case report

Table 1. Comparison of the characteristics of tract pain with radicular pain [6]

\begin{tabular}{cc}
\hline \multicolumn{1}{c}{ Tract Pain } & Radicular Pain \\
\hline Presented continuously or constantly & Exacerbated by stretching the root \\
\hline Symmetrical and non-dermatomal & Excellent localization \\
\hline Deep & Along dermatome \\
Hot or cold, burning, boring, aching, and superimposed sharp & Superficial \\
sensations & Sharp, stabbing \\
\hline Contralateral to cord compression & The same side as root compression \\
\hline Intense and does not correlate with physical findings & Correlates with physical findings \\
\hline
\end{tabular}




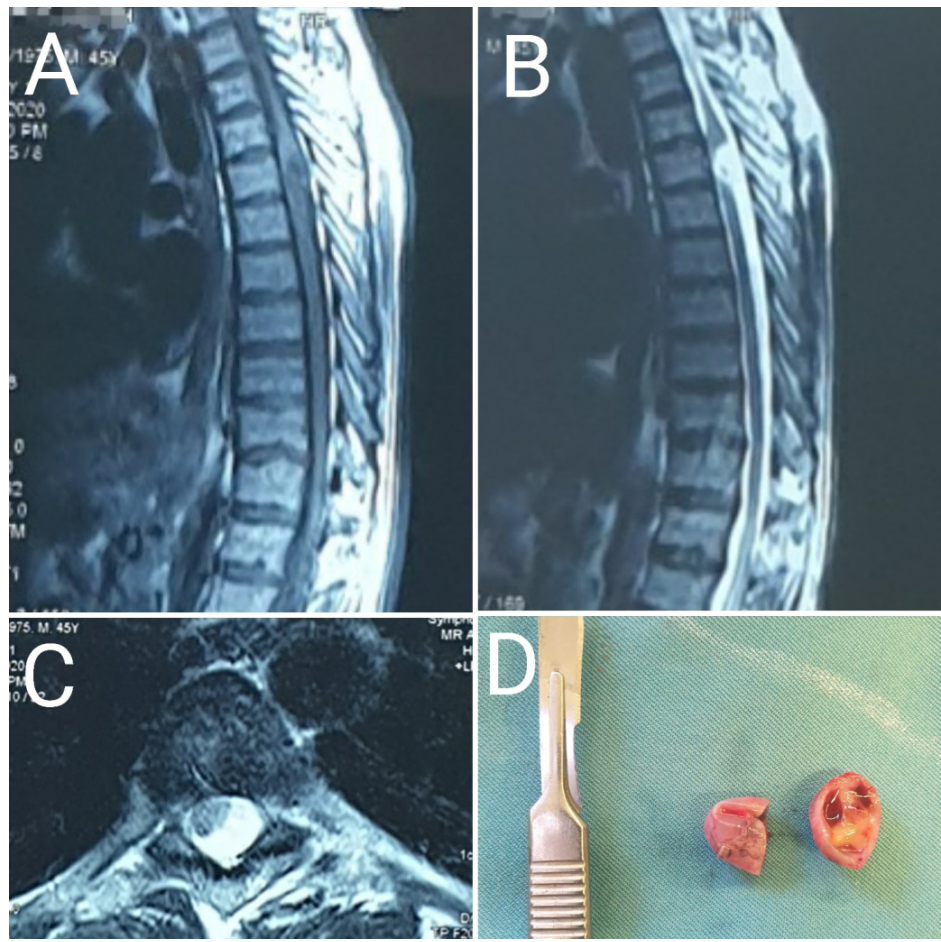

Orthopedic Science

Figure 1. Magnetic Resonance Imaging (MRI) and gross pathology findings of the Schwannoma

A and B: Tumor signal intensity on sagittal MRI, iso-signal on T1-WI (image A) and hyper signal with heterogeneity (cystic change) on T2-WI (Image B); C: Note the spinal cord deviation ventrolaterally due to the tumor compression; D: Cystic degeneration of tumor is seen in Schwannomas.

\section{Discussion}

This case is unusual because there was simulating clinical presentation of lumbar disc involvement; thus, the index surgeon treated the lumbar pathology first and did not search for more differential diagnoses and true etiology. Primary spinal cord tumors account for $2 \%-4 \%$ of all neoplasms of the central nervous system. Intradural extramedullary spinal cord tumors represent $60 \%-70 \%$ of all primary spinal cord tumors and are predominantly comprised of meningiomas, schwannoma, or neurofibroma [12]. Schwannomas are diagnosed typically in the fifth decade of life [13]. In a recent case series of intradural intramedullary tumors, the pain was the most common presenting symptom ( $72 \%)$ and manifested as back pain $(27 \%)$, radicular pain $(25 \%)$, or central pain $(20 \%)$. Motor disturbance was the next most common presenting symptom (55\%), followed by sensory loss (39\%) [14].

On MRI, Schwannomas appear as solid tumors in the dorsal sensory root region, with the displacement of the spinal cord. They are isointense on T1-WI and hyperintense on T2-WI [15]. In thoracic spinal cord compression, regardless of its etiology, unlike lumbar pathology, it can manifest unusual features, including neurogenic claudication with positionally dependent weakness, flaccid paraplegia, and even chronic abdominal pain $[1,3]$. The most common presentations of thoracic cord compression are midline back pain and myelopathy, but sciatica-like pain caused by thoracic cord compression due to the tumor, kyphosis, etc. has also been reported $[4,5]$.

Sciatica, a well-known marker of lumbar pathology can originate peripherally or centrally $[5,9]$. Lower thoracic disc (e.g., T10-11 and T11-12) herniation can compress lumbar enlargement of the spinal cord and the exiting nerve roots and brings about radiating pain through the lower extremity [1]. This finding was obtained by studying nerve roots organization from lumbar enlargement to conus medullaris, where caudal roots lie medially; therefore, a central compression from a disc or tumor or osteoligamentous complex can cause lower extremity pain or sciatica [16]. However, radiating pain originating from upper thoracic spine pathology needs further explanation. Kobayashi [17] delineated the concept of "Tract pain syndrome". The spinothalamic tract is known for its role in pain and temperature signal transmission. Any disruption or irritation of this tract could generate aberrant pain transmission and induce the feeling of a long tract pain (sciatica-like pain or burning pain). Mignucci 
Table 2. Data of the 21 reported patients

\begin{tabular}{|c|c|c|c|c|c|}
\hline \multicolumn{2}{|c|}{ Reference Case } & \multirow[t]{2}{*}{ Pathology } & \multirow{2}{*}{$\begin{array}{c}\text { Low Back Pain } \\
-\end{array}$} & \multirow{2}{*}{$\begin{array}{c}\begin{array}{c}\text { Straight Leg } \\
\text { Raising }\end{array} \\
\text { Slightly + }\end{array}$} & \multirow{2}{*}{$\begin{array}{c}\text { Exaggerated Deep Tendon } \\
\text { Reflex in Lower Extremity } \\
+\end{array}$} \\
\hline \multirow{6}{*}{ Scott [5] } & Case1 & & & & \\
\hline & Case2 & C1-C2 psammoma & - & NA & - \\
\hline & Case3 & T4 psammoma & - & NA & + \\
\hline & Case4 & T4 meningioma & + & NA & NA \\
\hline & Case5 & T2 neurofibroma & - & NA & NA \\
\hline & Case6 & T3 meningioma & - & NA & + \\
\hline \multirow{3}{*}{ Langfitt [18] } & Case1 & C4-C5 meningioma & + & Slightly + & NA \\
\hline & Case2 & C5-C6 disc herniation & + & NA & NA \\
\hline & Case3 & Cervical spondylosis & - & NA & NA \\
\hline \multicolumn{2}{|c|}{ Mignucci [9] } & T7-T8 disc herniation & Back pain & - & NA \\
\hline \multicolumn{2}{|c|}{ Brambilla et al. [29] } & C5-C6 osteophyte & + & NA & NA \\
\hline \multirow{2}{*}{ Ito [4] } & Case1 & C2-C3 tumor & - & - & - \\
\hline & Case2 & Thoracic kyphosis & + & - & - \\
\hline \multicolumn{2}{|c|}{ Neo [7] } & C3-C4 disc herniation & - & NA & - \\
\hline \multicolumn{2}{|c|}{ Ross [30] } & $\mathrm{C} 3-\mathrm{C} 4, \mathrm{C} 5-\mathrm{C} 6, \mathrm{C6}-\mathrm{C} 7$ & - & - & + \\
\hline \multirow{2}{*}{ Chan [6] } & Case1 & C3-C4,C4-C5 stenosis & - & NA & - \\
\hline & Case2 & C4-C5,C5-C6 stenosis & NA & NA & - \\
\hline \multirow{2}{*}{ Cho [3] } & Case1 & T6-T7 disc herniation & + & - & + \\
\hline & Case2 & T6-T7 disc herniation & + & - & + \\
\hline \multicolumn{2}{|c|}{ Yeung [28] } & C5-C6 disc herniation & + & NA & + \\
\hline \multicolumn{2}{|c|}{ Ganiüsmen [27] } & T10 menningioma & - & + & - \\
\hline
\end{tabular}

NA: Not available.
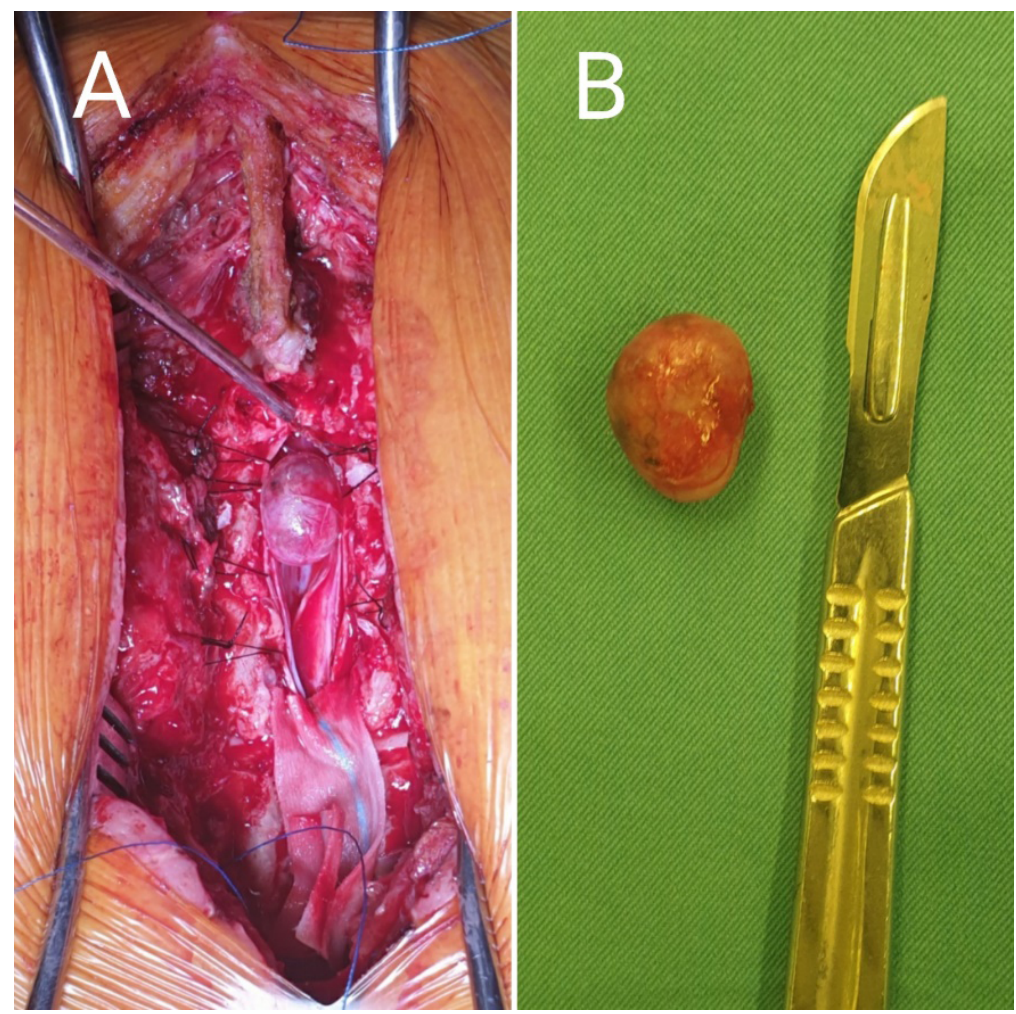

Figure 2. Macroscopic view of the Schwannoma tumor

A: Intraoperative view of the tumor after durotomy; B: Schwannoma tumor excised completely. 
[9] postulated that cord compression disinhibits normal ascending pain-producing spinal pathways that normally inhibit pain signals, which would permit the brain to interpret the lack of normal inhibition as pain.

The leg pain presentation is considered as 'false localizing' of cord compression, as there are discrepancies between the neurological signs and the expected anatomical location of the lesion. Besides, the absence of sharpness and precise localization challenges the diagnosis of a cord lesion [6]. To facilitate the diagnosis of "tract pain", some authors have attempted to characterize the pain as burning, boring, or aching compared with the sharp or stabbing nature of pain in nerve root disorder (Table 1) [18]. The pain that simulates sciatica is occasionally caused by contralateral cord compression. However, in the absence of a conclusive diagnostic key, pain relief after surgery is the only confirmation of "tract pain" [19].

Sciatica-like pain to the lower extremity due to cervical and thoracic spine pathology, including disc herniation and the tumoral lesion, has been reported in multiple studies [3-5, 9]. Slow-growing tumors, like benign tumors, lead to minimal symptoms as a result of the adjustment of the spinal cord; thus, the prevailing symptoms are radiculopathy, and sciatica-like pain caused by thoracic disc herniation and metastatic tumors results in more serious myelopathy and paralysis [20]. According to the anatomic distribution of lateral spinothalamic and corticospinal tract, cervical or thoracic cord-compressive lesions would commonly present with ipsilateral motor weakness and contralateral burning pain.

To our knowledge, 21 cases of sciatica caused by cervical and thoracic cord compression have been reported (Table 2). Spinal tumors, disc herniation, spondylosis, and long-lasting thoracic kyphosis are among the etiologies for sciatica tract pain. Vague signs and overlapping symptoms of thoracic spinal cord lesions can delay the correct diagnosis to a critical time when focal pathology has advanced to an irreversible state. The diagnosis of the thoracic spinal tumor was also difficult to make in our case because lumbar back pain and radiculopathy were the initial and major symptoms.

Our patient probably might have had upper motor neuron signs in his first examination, which had been overlooked; therefore, paying more attention to minor differences in jerk reflex, Babinski sign, and ankle clonus could have prevented him from reaching this advanced state of neurologic injury. Pathophysiology of sciatica-like pain in our patient can be the result of left side irritation of ascending spinothalamic tract through the pressure of tumoral mass at the T5 level, which caused more dominant contra-lateral (right side) back and leg pain. The patient's motor deficit can also be explained by disruption and irritation of the descending corticospinal tract.

The direct pressure on the neural elements not only could damage neurons but also could interfere with the vascular supply of the spinal cord, and resulted in peridural venous occlusions that in turn, could insult the already injured tissue. The anterior part of the thoracic spinal cord has relatively sparse arterial supplies and is more vulnerable to ischemia, which causes unexpected symptoms. This same scenario happened to our patient, his motor and bladder control rapidly declined over a course of two weeks that we considered vascular compromise responsible for the malignant deterioration, and our urgent surgery just could halt motor weakness from getting worse to complete paralysis. Even though the fair improvement was achieved one month postoperatively (muscle strength grade 1 to 3 ) but the bladder control still did not return to a voluntary state.

Similar to the countercoup effect on brain traumatology, the pressure generated by the compressive mass in a narrow thoracic spinal canal could have been transferred across the cord to affect the contralateral dorsal column and a corticospinal tract on the other side, causing symptomatology more confusing (e.g., a right side mass would generate both side symptoms) [20]. A high index of suspicion should be advised when symptoms do not match MRI findings, and cervical and thoracic spine MRI is worth ordering. Furthermore, the incidence of tandem spinal stenosis has been reported to range from 0.12 to $28 \%$ [21-23], this would be the rationale for search in different sections of the spine even when a definitive diagnosis of lumbar pathology has been made.

Other case studies have reported similar findings, for example, Rogers et al. [24] reported a 45-year-old man presenting with thoracic and lower back pain coincided with a minor back trauma, which persisted for a year and later revealed to be an Intradural Extramedullary (IE) Schwannoma at the T5 level on MRI examination. The patient was successfully treated with spinal surgery to remove a Schwannoma. Cox et al. [25] reported the case of a 30-year-old man presenting with lower right back and abdominal pain. MRI was ordered, and an IE tumor, specifically a Schwannoma at the T10 vertebral level, was discovered. The patient underwent total laminectomy of T10 and partial laminectomies of T9 and T11 to remove the tumor. Other spinal tumors could mimic the symptoms of Low Back Pain (LBP) as in Petersen et 
al. [19] and Osterhouse et al. [26] case reports, recurrent acute LBP revealed spinal tumors on MRI and CT to be a spinal myxopapillary ependymoma and ganglioneuroma, respectively. Yeung et al. covered the case of a 35-yearold man with neck pain and left-sided upper and lower extremity pain contralateral to herniated disc at the $\mathrm{C} 5$ to C6 level. All other spinal levels were normal without central canal stenosis or neural foraminal stenosis. An anterior cervical discectomy and fusion at the C5 to C6 level gave rise to complete resolution of his neck and left upper and lower extremity; thus, was attributed to tract pain.

\section{Conclusion}

Sciatica-like leg pain is a rare presentation of thoracic cord compression. It is postulated that the lateral spinothalamic tract could be involved due to cord compression by a thoracic Schwannoma tumor, resulting in predominantly contralateral symptoms that mimic a lumbar spine pathology. If the cause of pain in the lower extremity cannot be determined or does not match completely with clinico-anatomical consideration and lumbar MRI findings, there should be a low threshold for ordering thoracic and cervical MRI to examine the spinal cord. This case may provide further insights into other atypical presentations of thoracic spinal tumors. Also, the current idea that the pathologic spinal cord compression mass should necessarily cause ipsilateral symptoms is questioned by the case presented in this study.

\section{Ethical Considerations}

\section{Compliance with ethical guidelines}

All ethical principles are considered in this article. The participants were informed of the purpose of the research and its implementation stages. A written consent has been obtained from the subjects. principles of the Helsinki Convention was also observed.

\section{Funding}

This research did not receive any grant from funding agencies in the public, commercial, or non-profit sectors.

\section{Authors' contributions}

Conceptualization and Methodology: Khodakaram Rastegar; Data collection, Data analysis, and Writing original draft: Hasan Ghandhari and Farzam Mokarami; Writing - review \& editing: Ebrahim Ameri; Drafting the manuscript and data collection and literature review by Farzam Mokarami.

\section{Conflict of interest}

The authors declared no conflict of interest.

\section{Acknowledgments}

The authors would like to express their gratitude to Shafa hospital operating-room staffs for their support and providing me with all the facility that was required for this surgery

\section{References}

[1] Lyu RK, Chang HS, Tang LM, Chen ST. Thoracic disc herniation mimicking acute lumbar disc disease. Spine (Phila Pa 1976). 1999; 24(4):416-8. [DOI:10.1097/00007632-199902150 00025] [PMID]

[2] Oppenheim JS, Rothman AS, Sachdev VP: Thoracic herniated discs: Review of the literature and 12 cases. Mt Sinai J Med. 1993; 60(4):321-6. [PMID]

[3] Cho HL, Lee SH, Kim JS. Thoracic disc herniation manifesting as sciatica-like pain. Neurol Med Chir (Tokyo). 2011 51(1):67-71. [DOI:10.2176/nmc.51.67] [PMID]

[4] Ito T, Homma T, Uchiyama S. Sciatica caused by cervical and thoracic spinal cord compression. Spine (Phila Pa 1976). 1999; 24(12):1265-7. [DOI:10.1097/00007632-19990615000017] [PMID]

[5] Scott M. lower extremity pain simulating sciatica: Tumors of the high thoracic and cervical cord as causes. J Am Med Assoc. 1956; 160(7):528-34, [DOI:10.1001/ jama.1956.02960420008002] [PMID]

[6] Chan CH, Lee HY,Choi WC, Cho JY, Lee SH. Cervical cord compression presenting with sciatica-like leg pain. Eur Spine J. 2011; (suppl 2):S217-S221. [DOI:10.1007/s00586010-1585-5] [PMID] [PMCID]

[7] Neo M, Ido K, Sakamoto T, Matsushita M, Nakamura T. Cervical disc herniation causing localized ipsilateral popliteal pain. J Orthop Sci. 2002; 7(1):147-50 [DOI:10.1007/s776-0028437-2] [PMID]

[8] Demyer W. Pointers and pitfalls in the neurologic examination. Semin Neurol. 1998; 18(2):161-8. [PMID]

[9] Mignucci LA, Bell GR. Differential diagnosis of sciatica, in Rothman RH, Simeone FA(eds). Philadelphia: WB Saunders; 1992.

[10] Arce CA, Dohrmann GJ: Thoracic disc herniation: Improved diagnosis with computed tomographic scanning and a review of the literature. Surg Neurol. 1985; 23(4):356361. [DOI:10.1016/0090-3019(85)90206-X] [PMID]

[11] Balaqueá F, Fankhauser H, Rosazza A, Waldburge M Waldburger M: Unusual presentation of thoracic disc herniation. Clin Rheumatol. 1989; 8:269-73. [DOI:10.1007/ BF02030085] [PMID] 
[12] Grimm S, Chamberlain MC. Adult primary spinal cord tumors. Expert Rev. Neurother. 2009; 9(10):1487-95. [DOI:10.1586/ern.09.101] [PMID]

[13] Hirano K, Imagama S, Sato K, Kato F, Yukawa Y, Yoshihara $\mathrm{H}$, et al. Primary spinal cord tumors: Review of 678 surgically treated patients in Japan. A multicenter study. Eur Spine J. 2012; 21(10):2019-26. [DOI:10.1007/s00586-012-2345-5] [PMID] [PMCID]

[14] Raco A, Esposito V, Lenzi J, Piccirilli M, Delfini R, Cantore G. Long-term follow-up of intramedullary spinal cord tumors: A series of 202 cases. Neurosurgery. 2005; 56(5):972-981. [PMID]

[15] Abul-Kasim K, Thurnher MM, McKeever P, Sundgren PC. Intradural spinal tumors: Current classification and MRI features. Neuroradiology. 2008; 50(4):301-14. [DOI:10.1007/ s00234-007-0345-7] [PMID]

[16] Wall EJ, Cohen MS, Abitbol JJ, Garfin SR: Organization of intrathecal nerve roots at the level of the conus medullaris. J Bone Joint Surg Am. 1990; 72(10):1495-9. [DOI:10.2106/00004623-199072100-00010] [PMID]

[17] Kobayashi S."Tract pain syndrome" associated with chronic cervical disc herniation. Hawaii Med J. 1974; 33(10):376-81. [PMID]

[18] Langfitt TW, Elliot FA. Pain in the back and legs caused by cervical spinal cord compression. JAMA. 1967; 200(5):382-5. [PMID]

[19] Petersen D, Lystad RP. Spinal myxopapillary ependymoma in an adult male presenting with recurrent acute low back pain: A case report. Chiropr Man Therap. 2016; 24(11):1-5. [DOI:10.1186/s12998-016-0094-y] [PMID] [PMCID]

[20] Glasberg MR, Adornato BT. Disease of the spinal cord. In Rosenberg RN, editor. The Clinical Neurosciences. Neurology. New York: Churchill Livingstone; 1983.

[21] Laban MM, Green ML. Concurrent(tandem) cervical and lumbar spinal stenosis: A 10-yr review of 54 hospitalized patients. Am J Phys Med Rehabil. 2004; 83(3):187-90. [DOI:10.1097/01.PHM.0000113405.48879.45] [PMID]

[22] Luo CA, Kaliya-Perumal AK, Lu ML, Chen LH, Chen WJ, Niu CC. Staged surgery for tandem cervical and lumbar spinal stenosis: Which should be treated first? Eur Spine J. 2019; 28(1):61-8. [DOI:10.1007/s00586-018-5795-6] [PMID]

[23] Baker JF, MCh F. Evaluation and treatment of tandem spinal stenosis. J Am Acad Orthop Surg. 2020; 28(6):229-39. [DOI:10.5435/JAAOS-D-18-00726] [PMID]

[24] Rodgers AM, Khauv KB. Thoracic Schwannoma in an adult male presenting with thoracic pain: A case report. J Chiropr Med. 2017; 16(3):242-5. [DOI:10.1016/j.jcm.2017.02.002] [PMID] [PMCID]

[25] Cox JM, Alter M. Schwannoma: Challenging diagnosis. J Manipulative Physiol Ther. 2001; 24(8):526-8. [DOI:10.1067/ mmt.2001.118203] [PMID]

[26] Osterhouse MD, Kettner NW, Birrer PT, Mankamyer $\mathrm{K}$. Ganglioneuroma masquerading as spinal pain. J Manipulative Physiol Ther. 2002; 25(3):184-7. [DOI:10.1067/ mmt.2002.123335] [PMID]
[27] Ganiüsmen O, Coşkun S, Samancığlu A, Çıtak G. Thoracic spinal meningioma manifesting as Sciatica-like pain. J Nerv Syst Surg. 2014; 4(4):188-91. [DOI:10.5222/sscd.2014.188]

[28] Yeung JT, Johnson JI, Karim AS. Cervical disc herniation presenting with neck pain and contralateral symptoms: A case report. J Med Case Rep. 2012; 6:166. [DOI:10.1186/17521947-6-166] [PMID] [PMCID]

[29] Brambilla P, Monolo L, Giombelli E, Dorizzi A. Sciatica cordonale [Spinal cord sciatica]. Riv Neurobiol. 1984; 30(203):523-30. [PMID]

[30] Ross MD, Elliott R. Cervical cord compressive myelopathy in a man with a primary complaint of knee pain. J Spinal Cord Med. 2010; 33(4):431-4. [DOI:10.1080/10790268.2010. 11689724] [PMID] [PMCID] 
This Page Intentionally Left Blank 